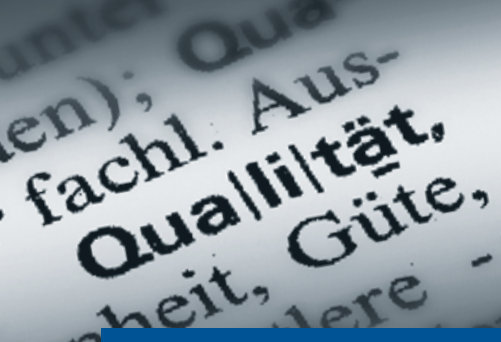

Heute stehen wir ...

... vor der Situation, dass

"Führung" immer noch ein eine Art Nachgedanke ist. Der beste Verkäufer wird Verkaufsleiter, der geübteste Techniker Betriebsleiter, der kenntnisreichste Zahlenjongleur Leiter des Controllings. Parallelen im Gesundheitswesen müssen nicht eigens erwähnt werden. Was sie für Führung brauchen, werden sich die Auserwählten schon irgendwie zulegen. Konfrontiert mit den Umbrüchen in der heutigen Arbeitswelt, etwa die ungebremste Pluralisierung der Wertvorstellungen und Motive, sollen sich Führungskräfte plötzlich mit dem Menschen als Subjekt und nicht als Objekt auseinandersetzen. Psychologie, Soziologie und Philosophie drängen sich auf. Überforderung macht sich breit.

H.K. Stahl

(aus: Führung als Profession,

Springer Medizin, 2021, in Druck)

\title{
Führungsbarometer Pflege 2021
}

\section{Ergebnisse zur Situation, Attraktivität und Bedeutung der Pflegeführung für die Zukunftsfähigkeit von Gesundheits- und Pflegeorganisationen}

In den vergangenen Monaten hatten Führungskräfte der Pflege verschärft durch die Corona-Pandemie einen raschen Wandel zu bewältigen. Dies nahm das Karl Landsteiner Institut für Human Factors \& Human Resources im Gesundheitswesen zum Anlass und führte im März und April mit EUCUSA, dem Spezialisten für Feedbacksysteme, eine Befragung durch, an der aus ganz Österreich annähernd 1.600 Führungskräfte teilnahmen. Dabei wurde der 2016 durchgeführte Führungsbarometer (vgl. Qualitas 04/16) in modifizierter Version wiederholt. Die Ergebnisse können österreichweit Grundlagen für zukünftige Maßnahmen und Strategien aus Sicht des Pflegemanagements und datenbasierte Informationen für Entscheidungsträger für gezielte Weiterentwicklung des Handlungsfeldes „Führung“ in der Pflege bieten.

Annelies Fitzgerald, Ursula Frohner, Lydia Viktoria Gromer, Barbara Klemensich, Inge KöberlHiebler, Manuel Kristler, Gabriele Thür, Alexander Seidl, Oliver Weichselbaumer, Sabine Wolf

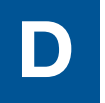

ie Ergebnisse zeichnen ein aktuelles Bild der Arbeitssituationen und unter welchen Voraussetzungen die Arbeit in der Pflegeführung als zufriedenstellend empfunden wird. Für den Wettbewerb am Arbeitsmarkt und für die Attraktivität des Berufsfeldes spielen auch veränderte Erwartungen von Mitarbeitern und Führungskräften eine bedeutende Rolle. Für die Sicherstellung der Zukunftsfähigkeit von Gesundheits- und Pflegeorganisationen erfordert dies eine verstärkte Einbeziehung in strategische Entscheidungen.

Speziell im Gesundheitswesen stellt Führung einen wesentlichen Erfolgsfaktor dar, um sowohl organisationsseitige als auch personenbezogene Entwicklungen zu erreichen. Positive Aspekte und Auswirkungen innerhalb der Organisation werden vielfach untersucht und beschrieben: Höhere Zufriedenheit, Commitment und Bindung von Mitarbeitern an die Organisation, stärker erlebte soziale Unterstützung durch Vorgesetzte und somit bessere Möglichkeiten, berufliche Belastungen - speziell bei Veränderungsprozessen und Fusionen - zu reduzieren, wirken sich im Sinne eines kybernetischen Regelkreises positiv auf 
die Betreuungsqualität aus (vgl. Schäfer 2021; Bakker \& Van Rhenen 2009).

\section{Was bewegt die Führungs- kräfte der Pflege 2021?}

Als positiv und wichtig wurde in der Befragung über alle Pflegebereiche und $\mathrm{Al}$ tersgruppen die Freude an den Kernaufgaben beurteilt. Als wesentlich werden ein konstruktives Teamklima und gegenseitige Wertschätzung formuliert (s. Abb 1).

Deutlichen Veränderungsbedarf sehen die Führungskräfte bei den zur Verfügung stehenden Ressourcen, um Pflege in hoher Qualität leisten zu können. Besonders kritisch werden Personalsituation, Entlohnung, mangelnde Einbindung in Entscheidungen und das Image der Pflegeberufe gesehen (s. Abb. 1, 3 und 4).
„Ausreichend Personal für den Regelbetrieb" hat für die Führungskräfte die höchste Bedeutung und wird von jungen Führungskräften am schlechtesten beurteilt. Die größte Diskrepanz in der Beurteilung der Einbindung in Entscheidungen, welche das Haus oder die Organisation betreffen, besteht zwischen dem Akut- (negativ) und dem Langzeitpflegebereich (positiv).

Überraschend und vertiefend zu untersuchen sind die Bedeutung der Akademisierung der Pflegeausbildung und die daraus resultierenden Veränderungen, die nur von Führungskräften mit einer akademischen Ausbildung leicht positiver eingeschätzt werden. Mehr als ein Drittel der befragten Führungskräfte gibt an, dass die Akademisierung der Pflegeausbildung keine positiven Veränderungen gebracht hat. Diese Einschätzung ist durchgängig, unabhängig von Alter, Jahren der Tätigkeit in der Führung, Bundesland, Träger oder Führungsebene (s. Abb. 2).

63\% der Befragungsteilnehmerinnen und -teilnehmer sehen positive nachhaltige Veränderungen im Team durch die Erfahrungen mit der COVID-19-Pandemie. Auch hier wäre genauer zu untersuchen, welche Faktoren dies begründen und inwiefern diese unabhängig von der Pandemie in Teams gestärkt werden können. Kein bedeutender Unterschied zwischen Altersgruppen zeigt sich bei der Einschätzung zum Entwicklungsbedarf. Dabei liegen die Schwerpunkte eher auf Soft Facts/Soft Skills, wie Stärkung der Eigenverantwortlichkeit oder Verbesserung der Kooperation. Die Tendenz zum verstärkten Einsatz von digitalen Lösungen zeigt sich in dieser Befragung eher gering.

Abb. 1: Das Wichtigste auf einen Blick

POSITIV:

- Freude an Arbeit (32)

- Teamklima (18)

- Wertschätzung durch MA (8)

- Identifikation als Führungskraft (2)

- Wertschätzung durch direkte Führungskraft (10)

- Für Führungsaufgaben gut gerüstet (1)

\section{NEGATIV:}

- Personalsituation (23)

- Image (13)

- Entlohnung (15)

- Ausreichend Ressourcen (24)

- Einbindung in Entscheidungen (7)

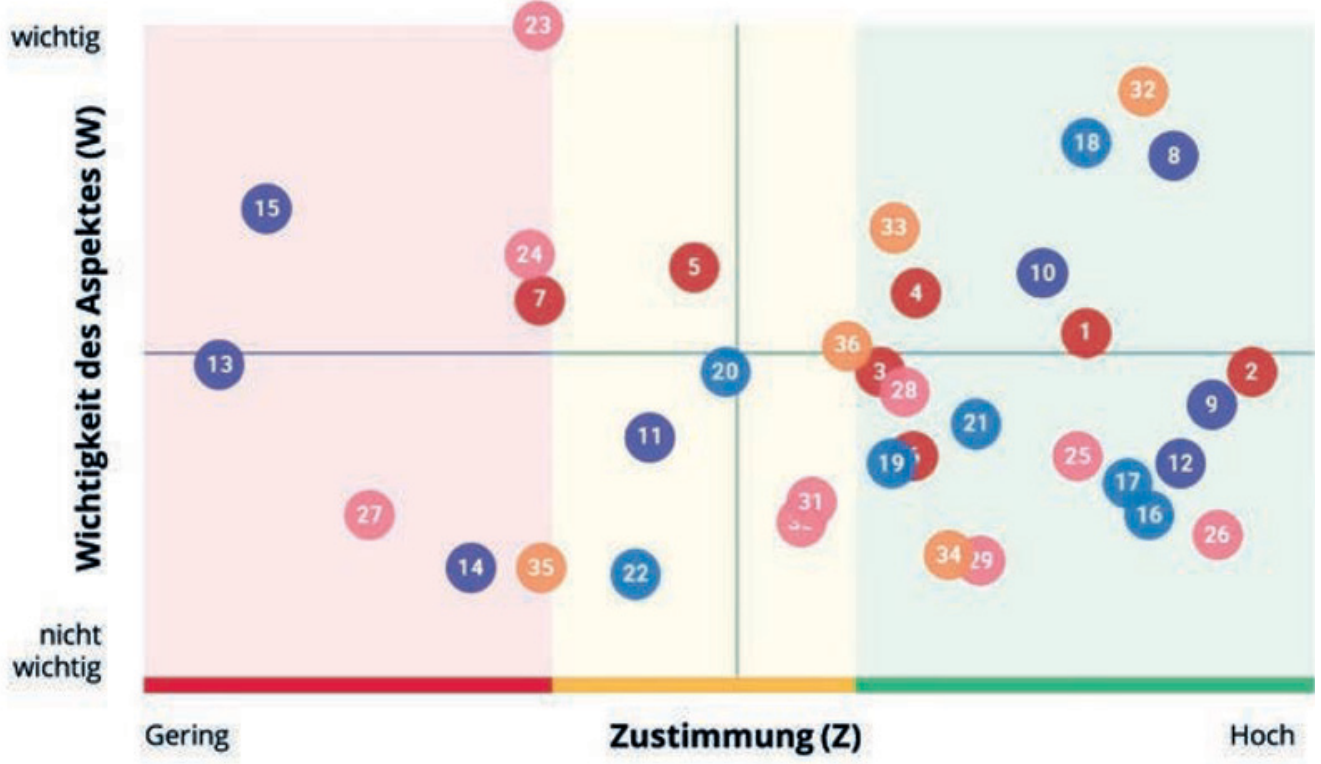

Die Lage der farbigen Punkte gibt Aufschluss über die Zustimmung zu den einzelnen Aspekten (Z liegt zwischen 0 - volle Ablehnung und 6 - volle Zustimmung) und deren Wichtigkeit (W liegt zwischen 0 und 100\%). 
Abb. 2: "Die Akademisierung der Pflege hat positive Veränderungen gebracht.“ 0 (Ablehnung der Aussage) bis 100 (Zustimmung zur Aussage)

\section{4}

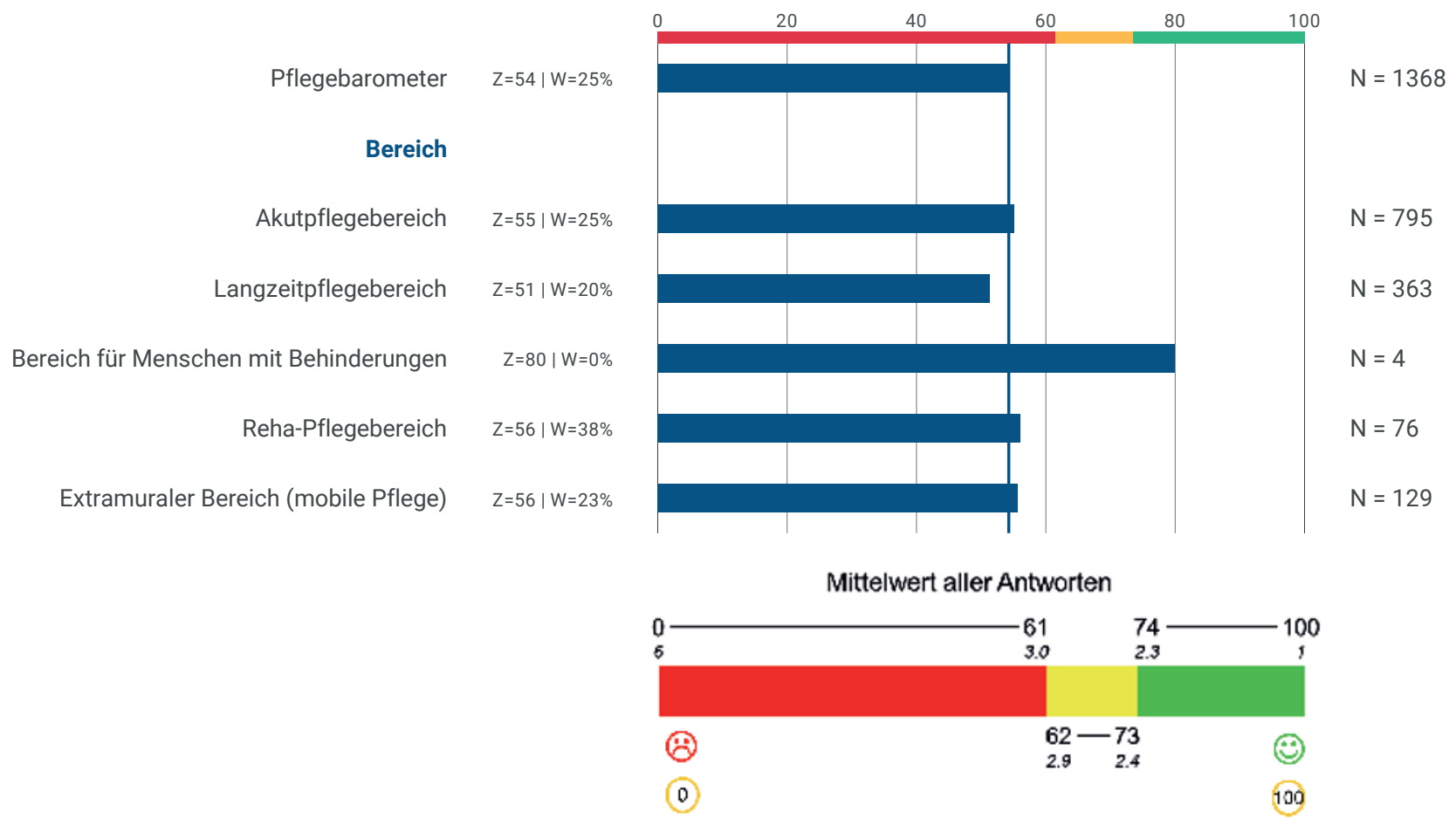

Abb. 3: "Wir haben für den Regelbetrieb ausreichend Personal“

0 (Ablehnung der Aussage) bis 100 (Zustimmung zur Aussage)

$\begin{array}{rr}\begin{array}{r}\text { Pflegebarometer } \\ \text { Wundesland }\end{array} & \mathrm{Z}=61 \mid \mathrm{W}=100 \% \\ \text { Niederösterreich } & \mathrm{Z}=57 \mid \mathrm{W}=100 \% \\ \text { Burgenland } & \mathrm{Z}=57 \mid \mathrm{W}=100 \% \\ \text { Oberösterreich } & \mathrm{Z}=65 \mid \mathrm{W}=100 \% \\ \text { Steiermark } & \mathrm{Z}=63 \mid \mathrm{W}=100 \% \\ \text { Kärnten } & \mathrm{Z}=71 \mid \mathrm{W}=100 \% \\ \text { Salzburg } & \mathrm{Z}=60 \mid \mathrm{W}=100 \% \\ \text { Tirol } & \mathrm{Z}=76 \mid \mathrm{W}=100 \% \\ \text { Vorarlberg } & \mathrm{Z}=64 \mid \mathrm{W}=98 \%\end{array}$

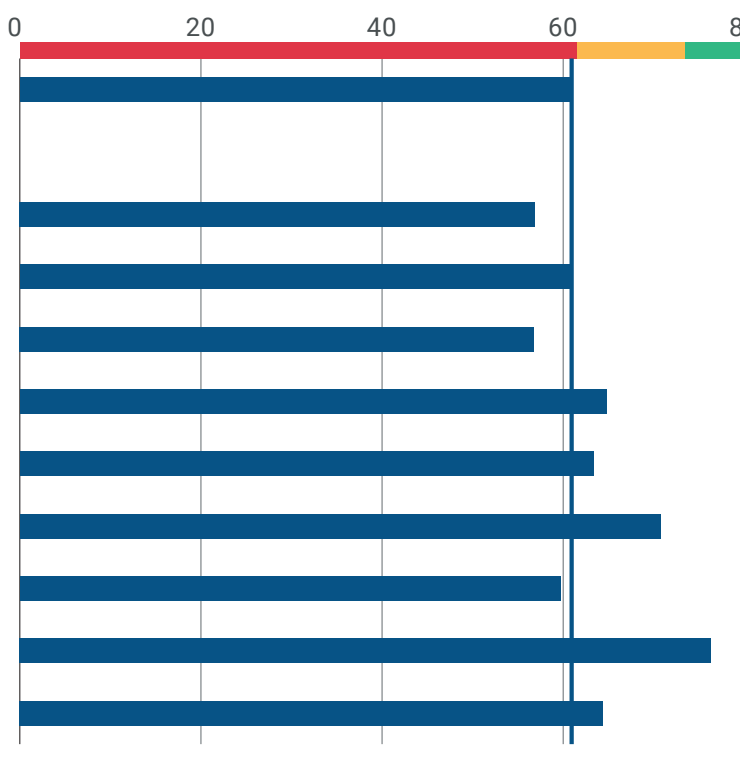

$N=1392$

$N=479$

$N=270$

$N=31$

$\mathrm{N}=84$

$N=221$

$\mathrm{N}=41$

$N=122$

$\mathrm{N}=38$

$\mathrm{N}=105$ 
Der Vergleich mit den Ergebnissen des Führungsbarometer Pflege 2016 zeigt, dass es die gleichen Aspekte sind, welche die Führungskräfte der Pflege zu ihrer Arbeit motivieren. Ressourcen sind dabei durchgängig Faktoren, welche den eigenen Gestaltungsspielraum, wie z.B. Zusammenarbeit im Team, betreffen. Es zeigt sich, dass die größten Probleme von 2016 fünf Jahre später unverändert aktuell sind, sich teilweise noch verschärft haben.

Nach wie vor gilt es, die berufspolitische Identität der Pflegeberufe insgesamt, welche einen wesentlichen Punkt in der Positionierung pflegerelevanter Themen darstellt, weiterzuentwickeln. Führungskräfte nehmen in der Gesamtversorgung eine bedeutende Rolle ein. Es liegt in ihrer Verantwortung, den kritischen gesundheits- und pflegepolitischen Diskurs zu führen, zu fördern und so die Bedeutung der Pflegeberufe gesamtgesellschaftlich zu unterstreichen.

\section{Träger- und organisations- übergreifende Vernetzung und Entwicklungsplanung}

Expertinnen und Experten verschiedener Träger, Organisationen und Einrichtungen aus allen Versorgungsbereichen haben es sich zum Ziel gesetzt, auf Basis der Ergebnisse sowie vertiefender Analysen, Maßnahmen zur Stärkung der bestehenden Ressourcen und der Steigerung der Attraktivität von Führungspositionen zu entwickeln und diese in gesundheitspolitische Prozesse und Gremien einzubringen (s. Tab. 1). In einer ersten Phase sind die Erarbeitung und Kommunikation eines nationalen Positionspapiers sowie eine Veranstaltung am 30.11.2021 zur Präsentation der Ergebnisse geplant.

Beiträge und Perspektiven von unterstützenden Organisationen und Mitgliedern der Expertengruppe finden Sie laufend auf www.kli-hr.at und auf den Social Media Seiten linkedin und facebook des Karl
Landsteiner Institutes für Human Factors \& Human Resources im Gesundheitswesen.

\section{Beispiele und Perspektiven aus den Organisationen der Expertengruppe}

\section{Lydia Viktoria Gromer, Pflegedirektorin der Barmherzigen Brüder Österreich:}

Seit Jahrzehnten begleitet das Management das Thema der Führungskräfteentwicklung, welche mit Blick auf die eigene Profession überaus vielfältig und in unterschiedlichen Formaten stattfindet.

Potenziale - erkennen und fördern: Potenzialerkennung und -förderung von Mitarbeitenden sind wichtige Säulen in der Gesamtentwicklung des Unternehmens. Dafür wird bei den Barmherzigen Brüdern das Mitarbeitergespräch als zentrales Instrument eingesetzt und alle Führungskräfte systematisch trainiert.

Rollen und Kompetenzen - stärken und gemeinsam wachsen: Für zukünftige Führungskräfte wurde ein standortübergreifender Lehrgang implementiert. Oberste Zielsetzung ist die Stärkung des Führungsverständnisses im Einklang mit den Werten sowie den Führungsgrundsätzen der Barmherzigen Brüder Österreich. Die multiprofessionelle und überregionale Zusammensetzung der Teilnehmenden ermöglicht es diesen, ein Netzwerk zwischen den Berufsgruppen und den Einrichtungen zu knüpfen. Eine großzügige Zeitplanung bietet Raum und Zeit, um im gegenseitigen Austausch die eigene Führungserfahrung sowie das eigene Führungsverständnis im Sinne der Ordenswerte zu entwickeln und zu reflektieren.

Teilnehmende berichten, dass sie durch diesen Lehrgang eine Stärkung erhalten und das gegenseitige, berufsgruppenübergreifende Verständnis unterstützt wird. Ebenso schätzen es die Teilnehmenden, abseits des Arbeitsalltags Zeit für die Reflexion von Führungsthemen zu haben.
Karriereentwicklung - der Orientierung Raum geben: Durch den enormen Wandel an die Anforderungen an Führungskräfte und die Gesamtentwicklung in der Pflege bekommt die Fachkarriere zunehmend höhere Bedeutung und drängt das Interesse an einer klassischen Führungskarriere in den Hintergrund. Wissend, dass das mittlere Management der Pflege in den Einrichtungen für alle relevanten Themen in der gesamten Versorgung die treibende Kraft bildet, ist das Ausbalancieren beider Perspektiven essenziell. Dabei hat die Orientierung in der Entscheidungsfindung hohe Relevanz. Für die Verknüpfung bzw. differenzierte Betrachtung von Führungsthemen und Fachentwicklung werden Potenzialträger mit Coachings unterstützt. Erwartet wird, dass die Investition in zukünftige Führungskräfte weiter steigen wird, um die Anforderungen an die Rolle erfüllen zu können. Dies wird bei allen strategischen Planungen mitberücksichtigt.

\section{Barbara Klemensich, Vinzenz Gruppe, Vorsitzende des Pflegemanagement- teams und strategische Leitung der Ausbildungsplattform:}

In Zeiten des Fachkräftemangels gewinnt der Ansatz des New Work und New Leadership an Bedeutung. Arbeit, Sinnhaftigkeit und Leben werden in neue Relationen zueinander gesetzt und schaffen neue Zusammenhänge. Die Unterschiedlichkeit der Generationen am Arbeitsmarkt hat das Zusammenleben in der Arbeit immer schon vor Herausforderungen gestellt, aber die veränderten digitalen Möglichkeiten, die unterschiedlichen Erwartungshaltungen potenzieren diese Herausforderungen. Wer bzgl. der künftigen Gestaltung der Arbeitswelt nach Standardlösungen fragt, wird wahrscheinlich nicht die gesuchte Lösung als Antwort erhalten.

Hilfreich sind nicht Patentrezepte, vielmehr sind bei der Umsetzung Kreativität und Mut gefragt. Es geht hier um die Frage, wie man sich diesem Kulturwandel stellen möchte oder muss. Eines ist sicher: Unternehmen, die sich nicht mit dem The- 
Abb. 4: Bei wichtigen, das Haus betreffende Themen und Entscheidungen werde ich als Führungskraft ausreichend miteingebunden. 0 (Ablehnung der Aussage) bis 100 (Zustimmung zur Aussage)

Pflegebarometer $\quad \mathrm{Z}=61 \mid \mathrm{W}=58 \%$

Bereich

$$
\text { Akutpflegebereich }
$$

Langzeitpflegebereich

Bereich für Menschen mit Behinderungen

Reha-Pflegebereich

Extramuraler Bereich (mobile Pflege)
$Z=52 \mid W=60 \%$

$Z=76 \mid W=54 \%$

$Z=52 \mid W=25 \%$

$Z=68 \mid W=62 \%$

$Z=68 \mid W=49 \%$

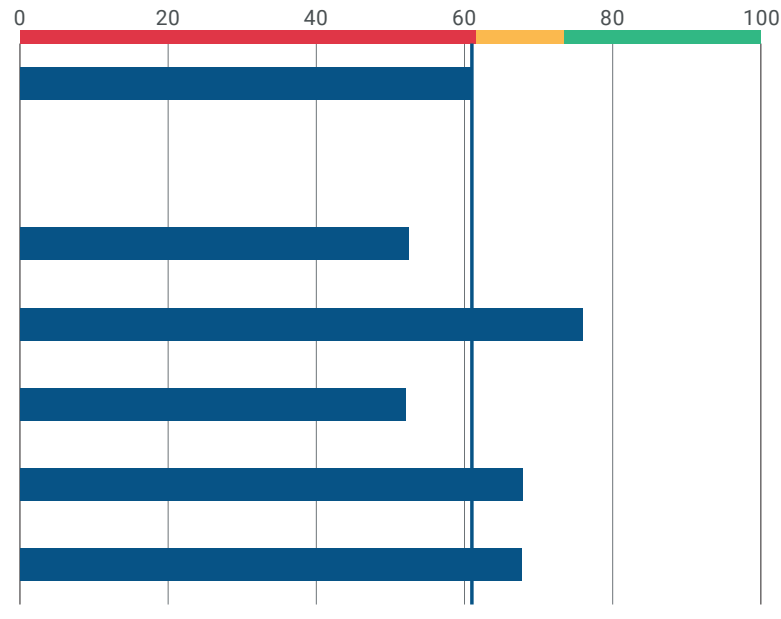

$N=1443$

$N=841$

$N=382$

$N=5$

$N=77$

$N=137$ ma New Work und New Leadership befassen und damit schlicht die Bedürfnisse ihrer Mitarbeiter ignorieren, setzen ihre Zukunftsfähigkeit aufs Spiel.

Unterschiedliche Altersgruppen bringen aufgrund ihrer unterschiedlichen Lebenssituationen verschiedene Erwartungen und Bedürfnisse mit. Deshalb ist für uns im Pflegemanagement Team in der Vinzenz Gruppe die Förderung der alter(n)sgerechten Führung Anliegen, Ziel und Erfolgsfaktor.

\section{Manuel Kristler, Sabine Wolf, Universitätsklinikum AKH Wien, Direktion des Pflegedienstes:}

Im Kontext der Führungskräfteentwicklung aber auch der Identifikation von "Young Talents“ in bestehenden Teams geht die Direktion des Pflegedienstes des AKH Wien verschiedene Wege. Neben gezielten Fortbildungen wurde als Tool für Nachwuchsführungskräfteentwicklung bzw. Nachfolgeplanung ein Kompetenzkatalog (nach Erpenbeck \& Heyse 2007), der „überfachliche“ Qualifikationen wie z.B. Lösungsorientierung oder Eigenverantwortung erfasst, entwickelt.

Die frühzeitige Identifizierung von potenziellen Nachwuchsführungskräften sowie deren Vorbereitung auf eine Leitungsfunktion ist eine wichtige Maßnahme zur Aufrechterhaltung der Führungsstrukturen. Um Talente im Haus zu entdecken, wurde auch ein direktions- und berufsgruppenübergreifendes Projekt initiiert: Mitarbeiterinnen und Mitarbeiter, die mittelfristig an einer Leitungsfunktion im Haus Interesse haben, sollen unkompliziert und niederschwellig angesprochen werden. Hierfür wird ein Online-Tool zur Selbsteinschätzung genutzt.

Ziel ist es, künftige Führungskräfte auf die vielfältigen Anforderungen optimal vorzubereiten und künftige wie bestehende Führungskräfte bei der Bewältigung ihrer Aufgaben und Pflichten zu unterstützen und sie im Sinne einer ergebniswirksamen Führung zu qualifizieren.
Gabriele Thür, Leiterin des Bereiches Gesundheits- und Krankenpflege, Wiener Gesundheitsverbund, Geriatriezentren und Pflegewohnhäuser der Stadt Wien:

In unseren Einrichtungen haben wir mit dem pflegerischen Organisationsmodell, aber auch mit Führungshandbüchern Teilerfolge erzielen können. So sind wir derzeit noch in der glücklichen Lage, mehrere Bewerberinnen und Bewerber für Führungspositionen zu haben.

Auszug aus dem Organisationsmodell zur Rolle als Teamverantwortliche: Das professionelle Fachwissen der DGKP im Langzeitbereich umfasst unter anderem eine konzeptionelle Auseinandersetzung und Praxisanwendung in der palliativen Grundversorgung, sowie die Anwendung komplementärer Pflegemethoden mit dem Ziel der Wahrung der Autonomie und der individuellen Lebensqualität der zu betreuenden Personen. Damit soll die Rolle der Führungskraft wieder in ihr eigenes Aufgabengebiet übergeführt werden. 
Tab. 1: Maßnahmengruppe Führungsbarometer Pflege 2021

\begin{tabular}{|c|c|c|}
\hline \multicolumn{3}{|l|}{ Leitung Maßnahmengruppe } \\
\hline \multicolumn{3}{|l|}{ Mitglieder Maßnahmengruppe } \\
\hline Tamara Archan, MSc,BScN & ÖGKV & 2. Vizepräsidentin \\
\hline Mag. Dr. Annelies Fitzgerald & $\begin{array}{l}\text { Karl Landsteiner Institut für Human Factors \& } \\
\text { Human Resources im Gesundheitswesen }\end{array}$ & Institutsleitung \\
\hline Lydia Viktoria Gromer, BA & Barmherzige Brüder Österreich & Pflegedirektorin der Ordensprovinz \\
\hline Doris Kazianka-Diensthuber, MAS & KABEG & Pflegedirektorin Gailtal-Klinik \\
\hline Barbara Klemensich, MBA & Vinzenz Gruppe & $\begin{array}{l}\text { Vorsitzende des Pflegemanagementteams und } \\
\text { strategische Leitung der Ausbildungsplattform }\end{array}$ \\
\hline Gabriele Thür & Wiener Gesundheitsverbund & Leiterin des Bereiches Pflege in der TU PWH \\
\hline Oliver Weichselbaumer & ÖGKV & Präsidiumsmitglied ÖGKV \\
\hline Sabine Wolf, MBA & Wiener Gesundheitsverbund & Pflegedirektorin AKH Wien \\
\hline
\end{tabular}

Führung ist ein ständiger und fortlaufender Prozess. Personalmanagement muss für Dienstleister die Königsdisziplin des Ressourcen-Management sein (vgl. Zulehner 2017).

Erforderliche Reformmaßnahmen wie die Festlegung der abzudeckenden Leistungsbereiche, die Verankerung von Ressourcenorientierung, Beziehungsarbeit und Personenorientierung als Grundleistungen und Arbeitsauftrag sowie einen Handlungsrahmen für den Pflegedienst sind gefordert. In der Pflege-Expertengruppe mitzuarbeiten erachte ich als sehr positiv - Erfahrungen einzubringen und damit die Zukunft der Pflege mitzugestalten.

\section{Oliver Weichselbaumer, Präsidiums- mitglied ÖGKV:}

Bereits die Initiative Führungsbarometer Pflege 2016 wurde vom Österreichischen Gesundheits- und Krankenpflegeverband (ÖGKV), damals unter der Präsidentschaft von Ursula Frohner, unterstützt. Wie Annelies Fitzgerald erklärt, ging es dabei nicht allein darum, Belastungsfaktoren zu untersuchen. Es sollten auch vorhandene Potenziale und Stärken identifiziert werden, auf welchen in Zukunft aufgebaut werden kann. Es brauche neue Weichenstellungen und radikal neue Denkmuster. Das ist nach wie vor aktuell.

Deshalb hält der ÖGKV auch unter der Präsidentschaft von Elisabeth Potzmann an der Unterstützung des Führungsbarometers Pflege fest. Unterstützt wird die Datengenerierung, auf Grundlage derer Maßnahmen geplant und umgesetzt werden können. Einen wichtigen Beitrag leistet der ÖGKV in seiner Akademie für Gesundheitsberufe, wo in einem Universitätslehrgang künftige Führungskräfte ausgebildet werden.

Zuletzt möchten wir noch auf den Abschlussbericht der Taskforce Pflege hinweisen (vgl. Ergebnisbericht Task Force Pflege, 2021). Präsidentin Potzmann leitete dabei eine Arbeitsgruppe, in welcher die Bedeutung der Fachkarrieren eingebracht wurden: Zusätzlich zu den klassi- schen Karrierewegen in der Pflege sollen künftig stärker Fachkarrieren ermöglicht werden, welche der Akademisierung der Pflege Rechnung tragen. Die Art, wie Pflegemanagement künftig gelebt wird, wird wesentlich zur Entwicklung des Pflegeberufes beitragen. Zentral dabei wird die Verweildauer im Beruf sein, wo aus Sicht des ÖGKV der Fokus auf ein erfolgreiches Onboarding gelegt werden muss. Dieses und andere Themen aus dem breiten Arbeitsspektrum der Führungskräfte werden in Gespräche mit Stakeholdern mitgenommen. Änderungen können und müssen gemeinsam in Angriff genommen werden.

Die ungekürzten Versionen finden Sie unter www.kli-hr.at.

Literatur bei den Autoren.

\section{Korrespondierende Autorin}

Mag. Dr. Annelies Fitzgerald

Leiterin des Karl Landsteiner Institut für Human Factors \& Human Resources

a.fitzgerald@karl-landsteiner.at

www.kli-hr.at 